\title{
Neck Circumference Is Better Associated Than Waist Circumference at Insulin Resistance in Bantu Population from Brazzaville in Republic of Congo
}

\author{
Danny Mafuta-Munganga ${ }^{1}$, Reine Freudlendrich Eboka-Loumingou Sakou ${ }^{2}$, \\ Benjamin Longo-Mbenza ${ }^{1,3,4}$, Etienne Mokondjimobe ${ }^{2,3}$, Blaise Makoso Nimi ${ }^{1,3,5}$ \\ ${ }^{1}$ Department of Internal Medicine, University of Kinshasa, Kinshasa, Democratic Republic of Congo \\ ${ }^{2}$ Faculty of Health Sciences, Marien Ngouabi University, Brazzaville, Republic of Congo \\ ${ }^{3}$ Department of Public Health, Lomo University of Research, Kinshasa, Democratic Republic of Congo \\ ${ }^{4}$ Faculty of Health Sciences, Walter Sisulu University, Mthatha, South Africa \\ ${ }^{5}$ Department of Internal Medecine, University of President Joseph KASA-VUBU, Boma, Democratic Republic of Congo
}

Email address:

danny.mafuta@gmail.com (D. Mafuta-Munganga), eliahdereine@gmail.com (R. F. Eboka-Loumingou S.), longombenza@gmail.com (B. Longo-Mbenza),mmobet@yahoo.fr (E. Mokondjimobe)

\section{To cite this article:}

Danny Mafuta-Munganga, Reine Freudlendrich Eboka-Loumingou Sakou, Benjamin Longo-Mbenza, Etienne Mokondjimobe, Blaise Makoso Nimi. Neck Circumference Is Better Associated Than Waist Circumference at Insulin Resistance in Bantu Population from Brazzaville in Republic of Congo. Science Journal of Clinical Medicine. Vol. 10, No. 1, 2021, pp. 7-11. doi: 10.11648/j.sjcm.20211001.12

Received: January 20, 2021; Accepted: February 14, 2021; Published: March 3, 2021

\begin{abstract}
Background: it is difficult to measure directly insulin sensitivity in routine practice. An easily measured anthropometric parameter associated with insulin resistance (IR) would be a very useful tool in detecting people at risk. Objective: To investigate the association between neck circumference (NC) and Insulin Resistance (IR) in Bantu population. Methods: Cross-sectional health screening conducted between February and May 2019, among Bantu population from Brazzaville in Republic of Congo. The analysis included 500 participants, aged $\geq 20$ years. Anthropometric indices and blood pressure were measured by standard protocol. Fasting lipid profile, blood glucose and insulin were determined. Triglyceride Glucose index (TyG index) and triglyceride/high density lipoprotein ratio (TG/HDL) were calculated. IR was defined as HOMA-IR $\geq 2$. TyG index was calculated by using the formula: $\mathrm{Ln}[\mathrm{TG}(\mathrm{mg} / \mathrm{dL}) \times \mathrm{FPG}(\mathrm{mg} / \mathrm{dL}) / 2]$. To investigate if there was a statistically association between NC and IR parameters, a correlation was computed. To evaluate the influence of NC on IR parameters, the study population was divided in 3 groups by tertiles of $\mathrm{NC}$ in both men and women The diagnostic ability of NC, WC, TyG index and TG/HDL ratio to identify people with IR was determined with the receiver operating characteristic (ROC) curves. Results: NC was positively correlated with insulin, TG/HDL, TyG index and HOMA-IR. For all variables, this correlation was stronger in comparison with WC. In all participants, TG/HDL shows the largest AUC for IR detection ( 0.810 , 95\% CI: $0.765-0.855)$ followed by TyG index $(0.799,95 \%$ CI: $0.754-0.844)$, NC $(0.624,95 \%$ CI: $0.572-0.677)$, and WC (0.616, 95\% CI: $0.560-0.672)$ in that order. Conclusion: NC is associated with IR. NC is better than WC to identify IR in Bantu population from Brazzaville in republic of Congo.
\end{abstract}

Keywords: Neck Circumference, Insulin Resistance, Bantu, Brazzaville

\section{Introduction}

Insulin resistance (IR) is considered as a major cardiometabolic risk factor. IR can be evaluated directly by invasive methods, including the hyperinsulinemiceuglycemic clamp technique [1-4]. These techniques are not accessible in current medical practice due to their invasiveness and their high cost for the vast majority of patients. Thus, several indexes have been proposed to indirectly measure cells insulin sensitivity. These are the assessment of the homeostasis model for insulin resistance (HOMA-IR) [5], the metabolic score for IR [6] and the triglyceride glucose index (TyG index) as well as TyG linked 
[7-9]. The dosage of insulin remains expensive to perform in current practice. It is for this reason; in the present study the objective is to evaluate the association between the neck circumference (NC), an anthropometric parameter easy to measure and the IR parameters (HOMA-IR, TyG index) in the Bantu population in sub-Saharan Africa.

\section{Methods}

\subsection{Study Population}

A cross-sectional survey on the diagnostic performance of the TG/HDL ratio in Insulin resistance in apparently healthy Congolese adults was done in Brazzaville. The details of the study design have been described previously [10]. In brief, the study was performed from February 14 to May 22, 2019. People (aged 20-80 years) who had lived in the community over 10 years, with informed consent were included. All participants under the age of 20, a known Diabetes mellitus (DM), with severe disabilities, hepatic failure, renal failure or goiter were excluded. A total of 500 participants were analyzed in this study.

\subsection{Ethical Consideration}

Written informed consents were obtained from all patients. All procedures were in accordance with the Helsinki Declaration of 1975, as revised in 2008.

\subsection{Clinical and Anthropometric Evaluation}

Waist circumference (WC) was measured using flexible tape between the highest lateral edge of the right and left Ilium. Neck circumference (NC) was measured in the middle of the neck between the mid-cervical spine and the midanterior neck at $0.5 \mathrm{~cm}$, so palpable, just below the laryngeal prominence. BMI was calculated as the weight in kilograms divided by the height in meters squared. Blood pressure (BP) was measured 3 times in a sitting position after at least 15 minutes of rest using an electronic type blood pressure monitor (OMRON M3 IT).

\subsection{Biochemical Measurements}

Peripheral venous blood samples were drawn after an overnight fast of at least $8 \mathrm{~h}$. The blood samples for the plasma glucose test were collected into vacuum tubes with the anticoagulant sodium fluoride and centrifuged within $1 \mathrm{~h}$ after collection. Plasma fasting concentrations of Glucose (FPG), Total Cholesterol (TC), Triglycerides (TG), high- density lipoprotein cholesterol (HDL) and Uric acid were measured using the standard procedure using a COBAS e411 (Roche Germany). Insulin was detected by the immunochemistry method.

\subsection{Definition of Variables}

1. Insulin resistance was estimated by HOMA-IR using the formula [5]: [Fast insulin $(\mathrm{mIU} / \mathrm{L}) \times$ FPG $(\mathrm{mmol} / \mathrm{L})] / 22.5$.

2. Insulin resistance was defined as a HOMA-IR $\geq 2.5$

3. The TyG index was calculated as the natural logarithm (Ln) of the product of FPG and TG using the formula [7]: TyG index $=\mathrm{Ln}[\mathrm{TG}(\mathrm{mg} / \mathrm{dL}) \times \mathrm{FPG}(\mathrm{md} / \mathrm{dL}) / 2]$

\subsection{Statistical Analysis}

Data analyses were performed with the software package SPSS Statistics, Version 21 (IBM Corporation, Armonk, NY, USA). Kolmogorov-Smirnov test of normality was used to determine the distribution of continuous variables. Normally distributed data were expressed as the means $\pm \mathrm{SD}$, whereas continuous variables with a skewed distribution were summarized as the median with interquartile range. Variables that are normally distributed were compared with independent sample t-test, whereas variables that are not normally distributed were compared with the Mann-Whitney test. Spearman's correlation coefficient was employed to test the correlations between different variables. The diagnostic ability of NC, WC, TyG index and TG/HDL ratio to identify people with IR was determined with the receiver operating characteristic (ROC) curves. A $P$ value $<0.05$ was considered statistically significant.

\section{Results}

Five hundred participants were enrolled. The mean of age was 47.2 years. The age, systolic blood pressure (SBP), diastolic blood pressure (DBP) and total cholesterol (TC) were similar in both IR and control groups. Table 1 shows generals characteristics of study population according to the IR status.

Table 2 demonstrated the correlation of $\mathrm{NC}$ and WC with IR parameters. To investigate if there was a statistically association between neck circumference and IR parameters, a correlation was computed. The NC was positively correlated with insulin, TG/HDL, TyG index and HOMA-IR in both males and females (all $p<0.05$ ). For all variables, this correlation was stronger in comparison with $\mathrm{WC}$.

Table 1. General characteristics of study participants by the presence of IR.

\begin{tabular}{|c|c|c|c|c|}
\hline Variables & All $(n=500)$ & IR (+) $(n=137)$ & IR (-) $(n=363)$ & $p$ \\
\hline Age, year & $47.2 \pm 13.6$ & $48.2 \pm 12.5$ & $46.8 \pm 14.1$ & 0.281 \\
\hline BMI, $\mathrm{kg} / \mathrm{m}^{2}$ & $26.4 \pm 5.1$ & $28.6 \pm 5.6$ & $25.6 \pm 4.6$ & $<0.001$ \\
\hline $\mathrm{NC}, \mathrm{cm}$ & $36.4 \pm 2.9$ & $37.4 \pm 2.7$ & $36.1 \pm 2.9$ & $<0.001$ \\
\hline $\mathrm{WC}, \mathrm{cm}$ & $93(85-103)$ & $98.0(89.5-105.0)$ & $93.0(85.0-101.0)$ & $<0.001$ \\
\hline $\mathrm{SBP}, \mathrm{mm} \mathrm{Hg}$ & $120(112-130)$ & $120(110-130)$ & $120(112-130)$ & 0.436 \\
\hline DBP, $\mathrm{mm} \mathrm{Hg}$ & $74(60-80)$ & $79(60-81)$ & $70(60-80)$ & 0.667 \\
\hline $\mathrm{TC}, \mathrm{mg} / \mathrm{dl}$ & $61.8(54.9-73.4)$ & $65.6(55.6-75.7)$ & $61.8(54.8-71.4)$ & 0.121 \\
\hline
\end{tabular}




\begin{tabular}{lllll}
\hline Variables & All $(\mathbf{n}=\mathbf{5 0 0})$ & IR $(+)(\mathbf{n}=\mathbf{1 3 7})$ & IR $(-)(\mathbf{n}=\mathbf{3 6 3})$ & $\boldsymbol{p}$ \\
\hline TG, $\mathrm{mg} / \mathrm{dl}$ & $133.2(108.0-155.8)$ & $153.1(122.1-167.3)$ & $122.1(100.9-154.0)$ & $<0.001$ \\
TG/HDL & $2.14(1.63-2.66)$ & $2.78(2.36-3.32)$ & $1.96(1.45-2.34)$ & $<0.001$ \\
FPG, $\mathrm{mg} / \mathrm{dl}$ & $84.0(76.0-98.0)$ & $99.0(81.0-151.5)$ & $82.0(74.0-93.0)$ & $<0.001$ \\
Insulin, UI/ml & $8.8(6.9-12.0)$ & $14.4(12.0-17.1)$ & $7.8(6.4-9.7)$ & $<0.001$ \\
HOMA-IR & $1.87(1.44-2.69)$ & $3.43(2.90-4.39)$ & $1.63(1.31-2.02)$ & $<0.001$ \\
TyG index & $8.64(8.37-8.88)$ & $8.91(8.70-9.30)$ & $8.54(8.26-8.75)$ & $<0.001$ \\
\hline
\end{tabular}

$I R+$ presence of insulin resistance, $I R$ - absence of insulin resistance, $B M I$ body mass index, $W C$ waist circumference, $N C$ neck circumference, $S B P$ systolic blood pressure, $D B P$ diastolic blood pressure, $F P G$ fasting plasma glucose, HOMA-IR insulin resistance index, $T G$ triglycerides, $T C$ total cholesterol, LDL low-density lipoprotein, $H D L$ high-density lipoprotein, $T y G$ triglyceride glucose index

Table 2. Correlation of IR parameters with neck and waist circumference stratified by gender.

\begin{tabular}{|c|c|c|c|c|c|c|c|c|}
\hline \multirow{3}{*}{ Variable } & \multicolumn{4}{|l|}{ Male } & \multicolumn{4}{|c|}{ Female } \\
\hline & \multicolumn{2}{|l|}{ NC } & \multicolumn{2}{|l|}{ WC } & \multicolumn{2}{|l|}{ NC } & \multicolumn{2}{|l|}{ WC } \\
\hline & $r_{s}$ & $p$-value & $r_{s}$ & $p$-value & $r_{s}$ & $p$-value & $r_{s}$ & $p$-value \\
\hline TG/HDL & 0.132 & 0.047 & 0.090 & 0.177 & 0.222 & $<0.001$ & 0.193 & 0.001 \\
\hline TyG index & 0.230 & 0.001 & 0.153 & 0.022 & 0.291 & $<0.001$ & 0.235 & $<0.001$ \\
\hline Insulin & 0.141 & 0.034 & 0.132 & 0.048 & 0.337 & $<0.001$ & 0.231 & $<0.001$ \\
\hline HOMA-IR & 0.228 & 0.001 & 0.157 & 0.019 & 0.395 & $<0.001$ & 0.247 & $<0.001$ \\
\hline
\end{tabular}

rs: Spearman coefficient

To evaluate the influence of NC on IR parameters, the study population was divided in 3 groups by tertiles of $\mathrm{NC}$ in both men and women. HOMA-IR and TyG index were significantly higher in the third tertile in comparison with the first and the second tertiles in men (table 3). However, the TG/HDL ratio, HOMA-IR, insulin and TyG index were significantly (all $p<0.001$ ) highest among the third tertile of $\mathrm{NC}$ in women (table 4).

Table 3. IR parameters according to the tertiles of neck circumference in men.

\begin{tabular}{|c|c|c|c|c|c|}
\hline Variables & All & $\mathrm{T} 1(<37.7 \mathrm{~cm})$ & T2 $(37.7-39.4 \mathrm{~cm})$ & T3 (> $39.4 \mathrm{~cm})$ & $p$ \\
\hline $\mathrm{N}$ & 225 & 75 & 76 & 74 & \\
\hline TG/HDL & $2.21(1.62-2.66)$ & $2.06(1.53-2.48)$ & $2.26(1.63-2.75)$ & $2.29(1.65-2.91)$ & 0.193 \\
\hline Insuline (UI/ml) & $8.6(6.5-11.7)$ & $7.5(6.2-11.0)$ & $8.7(6.8-12.0)$ & $9.0(6.7-12.8)$ & 0.189 \\
\hline HOMA-IR & $1.86(1.44-2.71)$ & $1.74(1.25-2.32)$ & $1.98(1.50-2.89)$ & $2.12(1.48-3.16)$ & 0.005 \\
\hline
\end{tabular}

Table 4. IR parameters according to the tertiles of neck circumference in women.

\begin{tabular}{|c|c|c|c|c|c|}
\hline Variables & All & $\mathrm{T} 1(<33.5 \mathrm{~cm})$ & T2 $(33.5-35.1 \mathrm{~cm})$ & T3 (> $35.1 \mathrm{~cm})$ & $p$ \\
\hline $\mathrm{N}$ & 275 & 91 & 93 & 91 & \\
\hline TG/HDL & $2.13(1.64-2.66)$ & $1.97(1.14-2.50)$ & $2.10(1.46-2.40)$ & $2.34(1.91-2.98)$ & $<0.001$ \\
\hline TyG index & $8.65(8.41-8.89)$ & $8.53(8.27-8.80)$ & $8.64(8.36-8.88)$ & $8.75(8.59-9.04)$ & $<0.001$ \\
\hline Insuline (UI/ml) & $9.1(7.1-12.3)$ & $8.1(6.4-10.6)$ & $8.4(7.0-10.7)$ & $12.0(8.5-16.5)$ & $<0.001$ \\
\hline HOMA-IR & $1.87(1.42-2.67)$ & $1.55(1.30-1.94)$ & $1.83(1.42-2.31)$ & $2.76(1.89-3.54)$ & $<0.001$ \\
\hline
\end{tabular}

Figure $1 \mathrm{~A}-\mathrm{C}$ shows the ROC curve analyses. Table 5 carries the corresponding AUCs $(95 \%$ confidence interval, CI). NC, WC, TG/HDL and TyG index significantly identified IR in all participants as well as in both genders. In all individuals, TG/HDL presents the largest AUC for IR identification $(0.810,95 \%$ CI: $0.765-0.855)$ followed by TyG index $(0.799,95 \%$ CI: $0.754-0.844), \mathrm{NC}(0.624,95 \% \mathrm{CI}$ : $0.572-0.677)$, and WC $(0.616,95 \%$ CI: $0.560-0.672)$ in that order. Gender analysis revealed that NC has large AUC than $\mathrm{WC}$ in both men and women, implying that it may better identify IR.

Table 5. Results of the AUC for NC, WC, TG/HDL and TyG index for IR identification

\begin{tabular}{|c|c|c|c|c|c|c|c|c|c|}
\hline \multirow{2}{*}{ Variables } & \multicolumn{3}{|l|}{ All } & \multicolumn{3}{|l|}{ Male } & \multicolumn{3}{|c|}{ Female } \\
\hline & AUC & $95 \%$ CI & $p$ & AUC & $95 \%$ CI & $p$ & AUC & $95 \%$ CI & $p$ \\
\hline $\mathrm{NC}, \mathrm{cm}$ & 0.624 & $0.572-0.677$ & $<0.001$ & 0.605 & $0.525-0.685$ & 0.015 & 0.727 & $0.656-0.797$ & $<0.001$ \\
\hline TG/HDL & 0.810 & $0.765-0.855$ & $<0.001$ & 0.774 & $0.700-0.849$ & $<0.001$ & 0.840 & $0.786-0.895$ & $<0.001$ \\
\hline TyG index & 0.799 & $0.754-0.844$ & $<0.001$ & 0.803 & $0.736-0.870$ & $<0.001$ & 0.796 & $0.736-0.857$ & $<0.001$ \\
\hline
\end{tabular}



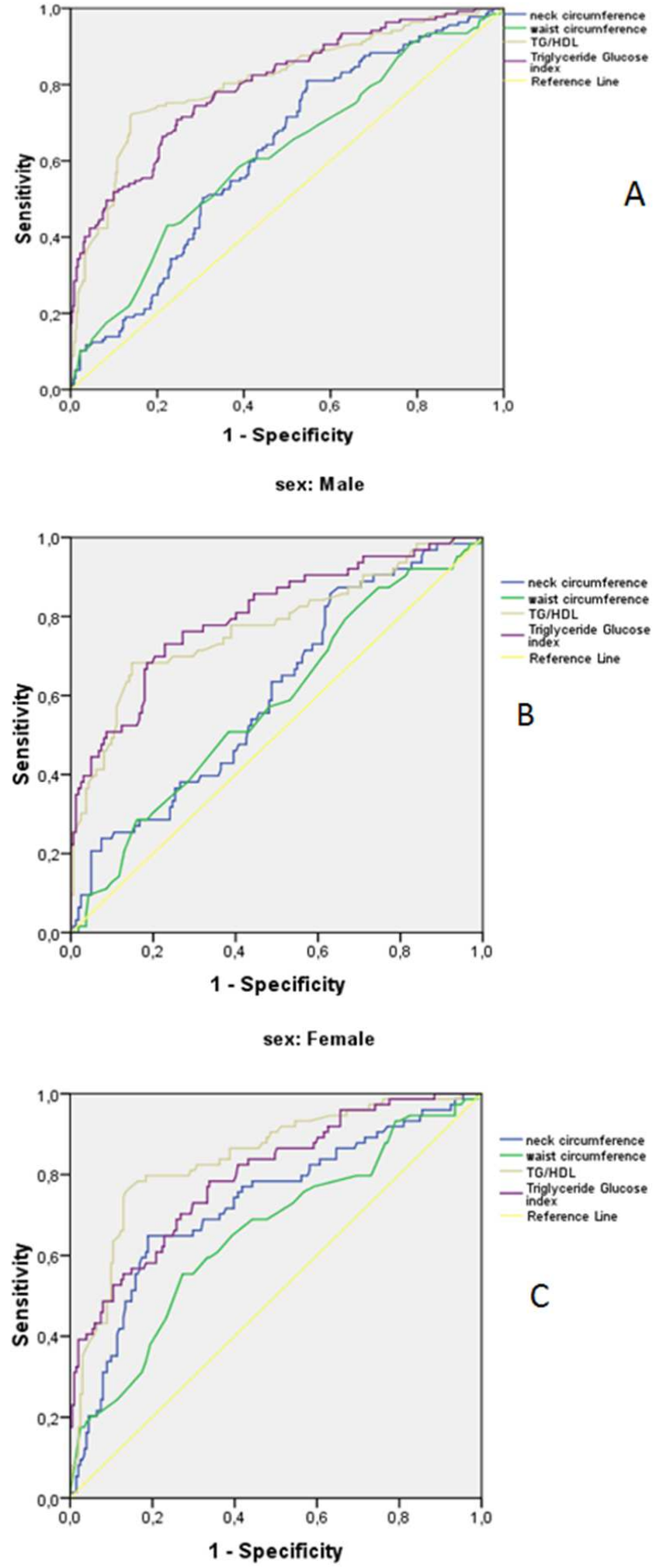

Figure 1. ROC curves for NC, WC, TG/HDL and TyG index for IR identification in all participants $(A)$, in men $(B)$ and in women $(C)$. IR insulin resistance; ROC, receiver operating characteristic; $T y G$, triglycerideglucose; TG/HDL triglyceride high density lipoprotein ratio.

\section{Discussion}

This study demonstrated that NC was associated with IR in
sub-Saharan Africa population.

However, in IR group, FPG, the fasting insulin, triglyceride, anthropometric indexes (NC, WC and $\mathrm{BMI})$ were highest in comparison with the control group. TG/HDL and TyG index, markers of IR were so higher among insulin resistant individuals than those in the control group. NC had a positive correlation with fasting insulin, TG/HDL ratio, TyG index and HOMA-IR; which corresponded well with previous studies [11]. In comparison with $\mathrm{WC}, \mathrm{NC}$ had the strongest correlation with IR parameters.

In type 2 Diabetes mellitus, IR is the primordial etiopathogenic mechanism $[12,13]$. Thus, individuals at risk must be identified. The invasive character of the glucose clamp technique to identify IR and the expensive measuring of insulin, limited their use in practical experience. Several studies proposed simple and non invasive methods [14 - 17]. Increased TyG index is commonly found in state of IR [7, 8, 18]. We proved that TyG index and HOMA-IR increased with the tertiles of NC.

In this study, the AUC was 0.624 (95\% CI: 0.572-0.677) for $\mathrm{NC}$ and $0.616(95 \% \mathrm{CI}: 0.560-0.672)$ for $\mathrm{WC}$ in predicting IR in all participants. Thus, that AUC for NC was higher than for $\mathrm{WC},(0.605$ [95\% CI: $0.525-0.685]$ versus 0.577 [95\% CI: $0.495-0.660])$ and $(0.727$ [95\% CI: $0.656-$ 0.797 ] versus 0.653 [95\% CI: $0.578-0.728]$ ), respectively in both men and women. This suggests that NC may be better than $\mathrm{WC}$ in predicting IR in Bantu population. Comparing to $\mathrm{WC}, \mathrm{NC}$ is an anthropometric measurement more efficient.

This study has the following limitation. By its crosssectional nature, associations between NC and IR parameters are not prospective and causal links cannot be inferred. The strength of the study lies in the fact that it is a large population-based study and representative of Brazzaville's adults. We describe, for the first time in Sub-Saharan region, the cross-sectional relationship of $\mathrm{NC}$ with insulin resistance.

\section{Conclusion}

This study verified the association between NC and IR in Bantu population from Brazzaville in republic of Congo. NC is better than WC to identify individuals with IR. Further research, particularly long-term, prospective, is needed in Central Africa region to validate our findings.

\section{References}

[1] DeFronzo RA, Tobin JD, Andres R. Glucose clamp technique: a method for quantifying insulin secretion and resistance. $\mathrm{Am}$ J Physiol 1979; 237: E214-23.

[2] Bergman RN, Prager R, Volund A, Olefsky JM. Equivalence of the insulin sensitivity index in man derived by the minimal model method and the euglycemic glucose clamp. $J$ Clin Invest 1987; 79: 790-800.

[3] Greenfield MS, Doberne L, Kraemer F, Tobey T, Reaven G. Assessment of insulin resistance with the insulin suppression test and the euglycemic clamp. Diabetes 1981; 30: 387-92. 
[4] Lim MH, Oh TJ, Choi K, Lee JC, Cho YM, Kim S. Application of the oral minimal model to Korean subjects with normal glucose tolerance and type 2 diabetes mellitus. Diabetes Metab J 2016; 40: 308-17.

[5] Galvin P, Ward G, Walters J, Pestell R, Koschmann M, Vaag A et al. A simple method for quantitation of insulin sensitivity and insulin release from an intravenous glucose tolerance test. Diabet Med 9: 921-928, 1992.

[6] Bello-Chavolla OY, Almeda-Valdes P, Gomez-Velasco D, Viveros-Ruiz T, Cruz-Bautista I, Romo-Romo A, et al. METS-IR, a novel score to evaluate insulin sensitivity, is predictive of visceral adiposity and incident type 2 diabetes. Eur J Endocrinol 2018; 178, 533-544. (https://doi.org/10.1530/EJE-17-0883)

[7] Simental-Mendia LE, Rodriguez-Moran M, Guerrero-Romero F. The product of fasting glucose and triglycerides as surrogate for identifying insulin resistance in apparently healthy subjects. Metab Syndr Relat Disord. 2008; 6: 299304. https://doi.org/10.1089/met.2008.0034

[8] Guerrero-Romero F, Simental-Mendia LE, Gonzalez-Ortiz M, Martınez-Abundis E, Ramos-Zavala MG, HernandezGonzalez SO, et al. The product of triglycerides and glucose, a simple measure of insulin sensitivity. Comparison with the euglycemic-hyperinsulinemic clamp. $J$ Clin Endocrinol Metab. 2010; 95: 3347-3351. https://doi.org/10.1210/jc.20100288

[9] Er L-K, Wu S, Chou H-H, Hsu L-A, Teng M-S, Sun Y-C, et al. (2016) Triglyceride Glucose-body Mass Index Is a Simple and Clinically useful Surrogate Marker for Insulin Resistance in nondiabetic Individuals. PLoS One 11 (3): e0149731. (doi: 10/ 1371/journal.pone.0149731).

[10] Eboka-Loumingou Sakou RF, Longo-Mbenza B, Guie G, MûnkaNkalla-Lambi A, Mokondjimobe E, Monabeka HG, Tukadila HA, Nkodila NA. Diagnostic Performance of the TG/HDL-C Ratio in the Prediction of Insulin Resistance in Brazzaville, Republic of Congo. International Journal of
Health Sciences and Research. Vol.10; Issue: 1; January 2020: $135-145$.

[11] Yang GR, Yuan SY, Fu HJ, Wan G, Zhu LX, Bu XL et al. Neck circumfence positively related with central obesity, overweight, and metabolic syndrome in Chinese subjects with type 2 diabetes: Beijing Community Diabetes Study 4 . Diabetes Care, 33 (2010), pp 2465-2467.

[12] Taylor R. Insulin resistance and type 2 diabetes. Diabetes. 2012; 61: 778-779. https://doi.org/10.2337/db12-0073

[13] Fonseca VA. Defining and characterizing the progression of type 2 diabetes. Diabetes Care. 2009; 32: S151-S156. https://doi.org/10.2337/dc09-S301

[14] Ren X, Chen ZA., Zheng S, Han T, Li Y, Liu W, et al. Association between triglyceride to HDL-C Ratio (TG/HDLC) and insulin resistance in Chinese patients with newly diagnosed type 2 diabetes mellitus. PLoS One. 2016; 11: e0154345. https://doi.org/10.1371/journal.pone.0154345

[15] Chiang JK, Lai NS, Chang JK, Koo M. Predicting insulin resistance using the triglyceride-to-high-density lipoprotein cholesterol ratio in Taiwanese adults. Cardiovasc Diabetol. 2011; 10: 93. https://doi.org/10.1186/1475-2840-10-93

[16] He J, He S, Liu K, Wang Y, Shi D, Chen X. The TG/HDL-C ratio might be a surrogate for insulin resistance in Chinese nonobese women. Int $J$ Endocrinol. 2014; 2014: 105168. https://doi.org/10.1155/2014/105168

[17] Kang HT, Yoon JH, Kim JY, Ahn SK, Linton JA, Koh SB, et al. The association between the ratio of triglyceride to HDL-C and insulin resistance according to waist circumference in a rural Korean population. Nutr Metab Cardiovasc Dis. 2012; 22: 1054-1060. https://doi.org/10.1016/j.numecd.2011.01.013

[18] Du T, Yuan G, Zhang M, Zhou X, Sun X, Yu X. Clinical usefulness of lipid ratios, visceral adiposity indicators, and the triglycerides and glucose index as risk markers of insulin resistance. Cardiovasc Diabetol. 2014; 13: 146. https://doi.org/10.1186/s12933-014-0146-3 